Proyecciones Journal of Mathematics

Vol. 33, No 1, pp. 205-213, June 2014.

Universidad Católica del Norte

Antofagasta - Chile

\title{
Strongly Bounded Partial Sums
}

\author{
Charles Swartz \\ New Mexico State University, U. S. A. \\ Received : February 2014. Accepted : April 2014
}

\begin{abstract}
If $\lambda$ is a scalar sequence space, a series $\sum z_{j}$ in a topological vector space $Z$ is $\lambda$ multiplier convergent in $Z$ if the series $\sum_{j=1}^{\infty} t_{j} z_{j}$ converges in $Z$ for every $t=\left\{t_{j}\right\} \in \lambda$. If $\lambda$ satisfies appropriate conditions, a series in a locally convex space $X$ which is $\lambda$ multiplier convergent in the weak topology is $\lambda$ multiplier convergent in the original topology of the space (the Orlicz-Pettis Theorem) but may fail to be $\lambda$ multiplier convergent in the strong topology of the space. However, we show under apprpriate conditions on the multiplier space $\lambda$ that the series will have strongly bounded partial sums.
\end{abstract}


The Orlicz-Pettis Theorem for locally convex spaces asserts that a series in such a space which is subseries convergent in the weak topology is subseries convergent in the Mackey topology and even in locally convex topologies which are stronger than the Mackey topology ([6], [9]). However, it is the case that a series in a locally convex space which is subseries convergent in the weak topology may fail to be convergent in the strong topology (see Example 1 below). Even though a series which is subseries convergent in the weak topology may fail to be convergent in the strong topology the partial sums of the series are bounded in the strong topology (see Theorem 3 below). If $\lambda$ is vector space of scalar valued sequences which contains $c_{00}$, the space of sequences which are eventually 0 , a series $\sum z_{j}$ in a topological vector space $(Z, \tau)$ is $\lambda$ multiplier convergent in $Z$ if the series $\sum_{j=1}^{\infty} t_{j} z_{j}$ is $\tau$ convergent for every $\left\{t_{j}\right\} \in \lambda$. For example, if $m_{0}$ is the sequence space of all scalars with finite range, then a series $\sum z_{j}$ is $m_{0}$ multiplier convergent iff the series is subseries convergent and if $\lambda=l^{\infty}$, then a series is $l^{\infty}$ multiplier convergent iff the series is bounded multiplier convergent. In this note we show that if the multiplier space $\lambda$ satisfies a gliding hump condition and $\sum x_{j}$ is a series in a locally convex space $X$ which is $\lambda$ multiplier convergent in the weak topology, then for every $t=\left\{t_{j}\right\} \in \lambda$ the partial sums of the series $\left\{\sum_{j=1}^{n} t_{j} x_{j}: n \in \mathbf{N}\right\}$ are strongly bounded.

We begin by fixing the notation and terminology. In what follows $X, X^{\prime}$ will denote a pair of vector spaces in duality; the weak topology on $X$ from $X^{\prime}$ will be denoted by $\sigma\left(X, X^{\prime}\right)$. The strong topology $\beta\left(X, X^{\prime}\right)$ on $X$ is the locally convex topology generated by the semi-norms

$$
p_{B}(x)=\sup \left\{\left|x^{\prime}(x)\right|: x^{\prime} \in B\right\}, x \in X,
$$

where $B$ runs over the family of all $\sigma\left(X^{\prime}, X\right)$ bounded subsets of $X^{\prime}$. See $[5],[11]$ for descriptions of the topologies.

Our results require a gliding hump assumption on the multiplier space $\lambda$. If $I \subset \mathbf{N}, \chi_{I}$ will denote the characteristic function of $I$ and if $t=\left\{t_{j}\right\}$ is scalar sequence, then $\chi_{I} t$ will denote the coordinatewise product of $t$ and $\chi_{I}$. If $m, n \in \mathbf{N}, m \leq n$, the interval $[m, n]$ is $\{j \in \mathbf{N}: m \leq j \leq n\}$; a sequence of intervals $\left\{I_{j}\right\}$ is increasing if $\max I_{j}<\min I_{j+1}$. A sign is a scalar $s_{j}= \pm 1$. The sequence space $\lambda$ has the signed weak gliding hump property (signed WGHP) if whenever $t=\left\{t_{j}\right\} \in \lambda$ and $\left\{I_{j}\right\}$ is an increasing sequence of intervals, there exist a subsequence $\left\{n_{j}\right\}$ and a sequence of signs $\left\{s_{j}\right\}$ such that the coordinate sum of the series $\sum_{j=1}^{\infty} s_{j} \chi_{I_{n_{j}}} t \in \lambda$; if all the signs $s_{j}$ can be chosen equal to $1, \lambda$ has the weak gliding hump 
property (WGHP). The WGHP was introduced by Noll ([7]) and the signed WGHP was introduced by Stuart ([8]) to treat questions of weak sequential completeness in $\beta$ duals. The space $\lambda$ is monotone if $\chi_{I} t \in \lambda$ whenever $t \in \lambda$ and $I \subset \mathbf{N}([1])$. A monotone space obviously has WGHP so spaces such as $c_{0}, l^{p}(o<p \leq \infty)$ have WGHP; the non-monotone space $c s$ of convergent series has WGHP and the space $b s$ of bounded series has signed WGHP but not WGHP ([8]). See [3] and [9] for further examples and [2] for descriptions of the sequence spaces.

The following example shows that a weakly subseries convergent series in a locally convex space may fail to be strongly subseries convergent.

Example 1. Consider the series $\sum e^{j}$ in $l^{\infty}$, where $e^{j}$ is the sequence with 1 in the $j^{\text {th }}$ coordinate and 0 in the other coordinates. The series is $\sigma\left(l^{\infty}, l^{1}\right)$ subseries convergent but not $\beta\left(l^{\infty}, l^{1}\right)=\|\cdot\|_{\infty}$ subseries convergent. Note, however, the partial sums of the series are $\|\cdot\|_{\infty}$ bounded.

Even though a weak subseries convergent series may fail to be strongly subseries convergent if the multiplier space $\lambda$ has signed WGHP, the partial sums of a weakly $\lambda$ multiplier convergent series has partial sums which are strongly bounded. In order to establish this result we require a matrix theorem due to Antosik and Mikusinski which we describe for the convenience of the reader.

Theorem 2. (Antosik-Mikusinski) Let $Z$ be a topological vector space and $x_{i j} \in Z$ for $i, j \in \mathbf{N}$. Suppose (I) $\lim _{i} x_{i j}=x_{j}$ exists for every $j$ and (II) for each increasing sequence of positive integers $\left\{m_{j}\right\}$ there exist a subsequence $\left\{n_{j}\right\}$ of $\left\{m_{j}\right\}$ and a sequence of signs $\left\{s_{j}\right\}$ such that $\left\{\sum_{j=1}^{\infty} s_{j} x_{i n_{j}}\right\}_{i=1}^{\infty}$ converges. Then $\lim _{i} x_{i i}=0$.

More general forms of the theorem can be found in Appendix D of [9]. An infinite matrix $\left[x_{i j}\right]$ satisfying conditions (I) and (II) is called a signed $\mathcal{K}$ matrix. The signed version of the Antosik-Mikusinski Matrix Theorem is due to Stuart ([8]).

Theorem 3. Suppose $\lambda$ has signed WGHP and the series $\sum x_{j}$ is $\lambda$ multiplier convergent with respect to $\sigma\left(X, X^{\prime}\right)$. Then for every $t=\left\{t_{j}\right\} \in \lambda$ the partial sums $\left\{\sum_{j=1}^{n} t_{j} x_{j}: n \in \mathbf{N}\right\}$ are $\beta\left(X, X^{\prime}\right)$ bounded. 
Proof. Suppose the conclusion fails. Then there exist a $\sigma\left(X^{\prime}, X\right)$ bounded set $B$, an increasing sequence $\left\{n_{k}\right\}, \epsilon_{k} \rightarrow 0, \epsilon_{k}>0$, and $\delta>0$ such that

$$
\epsilon_{k} p_{B}\left(\sum_{j=1}^{n_{k}} t_{j} x_{j}\right)>\delta
$$

for every $k$. For $k=1$ we have $p_{B}\left(\epsilon_{1} \sum_{j=1}^{n_{1}} t_{j} x_{j}\right)>\delta$ so there exists $x_{1}^{\prime} \in B$ such that $\epsilon_{1}\left|x_{1}^{\prime}\left(\sum_{j=1}^{n_{1}} t_{j} x_{j}\right)\right|>\delta$. There exists $l_{1}$ such that $l \geq l_{1}$ implies

$$
\text { (\#) } \epsilon_{l} p_{B}\left(\sum_{j=1}^{n_{1}} t_{j} x_{j}\right)<\delta / 2 \text {. }
$$

Put $k_{1}=1$ and $k_{2}=\max \left\{l_{1}, k_{1}\right\}$ so $\epsilon_{k_{2}} p_{B}\left(\sum_{j=1}^{n_{k_{2}}} t_{j} x_{j}\right)>\delta$ and there exists $x_{2}^{\prime} \in B$ such that

$$
\epsilon_{k_{2}}\left|x_{2}^{\prime}\left(\sum_{j=1}^{n_{k_{2}}} t_{j} x_{j}\right)\right|>\delta
$$

Then from (\#),

$\epsilon_{k_{2}}\left|x_{2}^{\prime}\left(\sum_{j=n_{k_{1}+1}}^{n_{k_{2}}} t_{j} x_{j}\right)\right| \geq \epsilon_{k_{2}}\left|x_{2}^{\prime}\left(\sum_{j=1}^{n_{k_{2}}} t_{j} x_{j}\right)\right|-\epsilon_{k_{2}}\left|x_{2}^{\prime}\left(\sum_{j=1}^{n_{k_{1}}} t_{j} x_{j}\right)\right|>\delta-\delta / 2=\delta / 2$.

Continuing this construction produces an increasing sequence $\left\{k_{p}\right\},\left\{x_{p}^{\prime}\right\} \subset$ $B$ such that if $\left[n_{k_{p-1}+1}, n_{k_{p}}\right]=I_{p}$, then

$$
\text { (*) } \epsilon_{k_{p}}\left|x_{p}^{\prime}\left(\sum_{j \in I_{p}} t_{j} x_{j}\right)\right|>\delta / 2
$$

Define an infinite matrix

$$
M=\left[m_{p q}\right]=\left[\epsilon_{k_{p}} x_{p}^{\prime}\left(\sum_{j \in I_{q}} t_{j} x_{j}\right)\right] .
$$

We claim that $M$ is a signed- $\mathcal{K}$ matrix. First, the columns of $M$ converge to 0 since $\left\{x_{p}^{\prime}\right\}$ is $\sigma\left(X^{\prime}, X\right)$ bounded and $\epsilon_{k} \rightarrow 0$. Next, given any increasing sequence of integers, by the signed WGHP property there exist a further subsequence $\left\{r_{q}\right\}$ and signs $\left\{s_{q}\right\}$ such that $u=\sum_{q=1}^{\infty} s_{q} \chi_{I_{r_{q}}} t \in \lambda$. Then

$$
\sum_{q=1}^{\infty} s_{q} m_{p r_{q}}=\sum_{q=1}^{\infty} \epsilon_{k_{p}} x_{p}^{\prime}\left(s_{q} \sum_{j \in I_{r_{q}}} t_{j} x_{j}\right)=\epsilon_{k_{p}} x_{p}^{\prime}\left(\sum_{q=1}^{\infty} s_{q} \sum_{j \in I_{r_{q}}} t_{j} x_{j}\right)=\epsilon_{k_{p}} x_{p}^{\prime}\left(\sum_{q=1}^{\infty} u_{q} x_{q}\right),
$$


where $\sum_{q=1}^{\infty} u_{q} x_{q}$ is the $\sigma\left(X, X^{\prime}\right)$ sum of the series. Then $\lim _{p} \sum_{q=1}^{\infty} s_{q} m_{p r_{q}}=$ 0 since $\epsilon_{p} \rightarrow 0$ and $\left\{x_{p}^{\prime}\right\}$ is $\sigma\left(X, X^{\prime}\right)$ bounded. By the Antosik-Mikusinski Matrix Theorem, the diagonal of $M$ converges to 0 . But, this contradicts $(*)$ and the result follows.

A similar result under different assumptions was established in $[9,2.6]$; the result above requires no topology on the multiplier space (e.g., the spaces $d$ and $\delta$ ([4, pages 48 and 68]). In particular, the theorem applies to subseries and bounded multplier convergent series. It should be pointed out that the series $\sum x_{j}$ in Theorem 3 is actually $\lambda$ multiplier convergent in the Mackey topology since it is assumed that $\lambda$ has signed WGHP (see the Orlicz-Pettis Theorem 4.10 of [9]).

The following example shows that without some assumption on the multiplier space $\lambda$ the conclusion of Theorem 3 may fail to hold. Let $\mu$ be the scalar sequence space of all sequences which are eventually constant. In this case a series $\sum z_{j}$ in a topological vector space $(Z, \tau)$ is $\mu$ multiplier convergent with respect to $\tau$ iff the series is $\tau$ convergent.

Example 4. Let $c_{00}$ be the space of all real sequences which are eventually 0 with the sup norm $\|\cdot\|_{\infty}$. The dual of this space is $l^{1}$. The sequence $\left\{j e^{j}\right\}$ is $\sigma\left(l^{1}, c_{00}\right)$ convergent to 0 since if $t=\left\{t_{j}\right\} \in c_{00}$, then $j e^{j}(t)=0$ eventually. Set $e^{0}=0$. Then the telescoping series $\sum_{j=1}^{\infty}\left(j e^{j}-(j-1) e^{j-1}\right)$ is $\sigma\left(l^{1}, c_{00}\right)$ convergent to 0 and is, therefore, $\mu$ multiplier convergent with respect to $\sigma\left(l^{1}, c_{00}\right)$. However, $\beta\left(l^{1}, c_{00}\right)=\|\cdot\|_{1}$ and if $u=\{1\} \in \mu$, then

$$
\left\|\sum_{j=1}^{n} u_{j}\left(j e^{j}-(j-1) e^{j-1}\right)\right\|_{1}=\left\|n e^{n}\right\|_{1}=n
$$

so the partial sums of the series are not strongly bounded.

If the multiplier space $\lambda$ satisfies a stronger gliding hump assumption and the space $X$ satisfies an additional condition, the conclusion of Theorem 3 can be strengthened.

The space $\lambda$ is a K-space if $\lambda$ has a Hausdorff vector topology under which the coordinate maps $t=\left\{t_{j}\right\} \rightarrow t_{j}$ are continuous from $\lambda$ into the scalar field. Let $\mathcal{F}$ be a family of subsets of $\lambda$. The K-space $\lambda$ has the signed $\mathcal{F}$ gliding hump property (signed $\mathcal{F}$ GHP) if for every $F \in$ $\mathcal{F}$ whenever $\left\{t^{k}\right\} \subset F$ is bounded and $\left\{I_{k}\right\}$ is an increasing sequence of intervals, there exist subsequence $\left\{n_{k}\right\}$ and a sequence of signs $\left\{s_{k}\right\}$ such that the coordinate sum of the series $\sum_{k=1}^{\infty} s_{k} \chi_{I_{n_{k}}} t^{k}$ belongs to $\lambda$; if all the signs can be chosen equal to 1 , then $\lambda$ has the $\mathcal{F}$ gliding hump property 
$(\mathcal{F}$ GHP). For example, if $\mathcal{F}$ consists of all the finite subsets of $\lambda$, signed $\mathcal{F}$ GHP $(\mathcal{F}$ GHP) is just the signed WGHP (WGHP) and if $\mathcal{F}$ consists of all the bounded subsets of $\lambda$, signed $\mathcal{F}$ GHP $(\mathcal{F}$ GHP) corresponds to the signed strong gliding hump property (strong gliding hump property). The spaces $l^{\infty}$ and $m_{0}$ have the strong gliding hump property while the space bs has the signed strong gliding hump property but not the strong gliding hump property. For other spaces with the $\mathcal{F}$ GHP see [10]. For further examples of spaces with the strong gliding hump property, see Appendix B of [9] and [3].

For the result we require the following observation. Recall a locally convex space $X$ is a Banach-Mackey space if bounded subsets of $X$ are strongly bounded $([11,10.4 .3])$. For example, any barrelled space is a Banach-Mackey space since it carries the strong topology while every sequentially complete locally convex space is a Banach-Mackey space $([11,10.4 .8])$.

Lemma 5. Assume $X$ is a Banach-Mackey space and $\lambda$ is a $K$-space. If $A \subset \lambda$ is bounded and $B \subset X^{\prime}$ is $\sigma\left(X^{\prime}, X\right)$ bounded, then for every $x \in X$, $k \in \mathbf{N}$,

$$
p_{B}\left(\left\{t_{k} x: t=\left\{t_{j}\right\} \in A\right\}\right)<\infty .
$$

Proof. Since $\lambda$ is a K-space, the set $\left\{t_{k} x: t=\left\{t_{j}\right\} \in A\right\}$ is bounded in $X$ and, therefore, strongly bounded by the Banach-Mackey assumption.

Theorem 6. Suppose $X$ is a Banach-Mackey space, $\lambda$ has signed $\mathcal{F}$ GHP and the series $\sum x_{j}$ is $\lambda$ multiplier convergent with respect to $\sigma\left(X, X^{\prime}\right)$. Then for every bounded subset $A \in \mathcal{F}$ the partial sums $\left\{\sum_{j=1}^{n} t_{j} x_{j}: t \in\right.$ $A, n \in \mathbf{N}\}$ are $\beta\left(X, X^{\prime}\right)$ bounded.

Proof. Suppose the conclusion fails. Then there exist a $\sigma\left(X^{\prime}, X\right)$ bounded set $B,\left\{t^{k}\right\} \subset A$, an increasing sequence $\left\{n_{k}\right\}, \epsilon_{k} \rightarrow 0, \epsilon_{k}>0$, and $\delta>0$ such that

$$
\epsilon_{k} p_{B}\left(\sum_{j=1}^{n_{k}} t_{j}^{k} x_{j}\right)>\delta
$$

for every $k$. For $k=1$ we have $p_{B}\left(\epsilon_{1} \sum_{j=1}^{n_{1}} t_{j}^{1} x_{j}\right)>\delta$ so there exists $x_{1}^{\prime} \in B$ such that $\epsilon_{1}\left|x_{1}^{\prime}\left(\sum_{j=1}^{n_{1}} t_{j}^{1} x_{j}\right)\right|>\delta$. By the lemma there exists $l_{1}$ such that $l \geq l_{1}$ implies

$$
\text { (\#) } \epsilon_{l} p_{B}\left(\sum_{j=1}^{n_{1}} t_{j}^{l} x_{j}\right)<\delta / 2 \text {. }
$$


Put $k_{1}=1$ and $k_{2}=\max \left\{l_{1}, k_{1}\right\}$ so $\epsilon_{k_{2}} p_{B}\left(\sum_{j=1}^{n_{k_{2}}} t_{j}^{k_{2}} x_{j}\right)>\delta$ and there exists $x_{2}^{\prime} \in B$ such that

$$
\epsilon_{k_{2}}\left|x_{2}^{\prime}\left(\sum_{j=1}^{n_{k_{2}}} t_{j}^{k_{2}} x_{j}\right)\right|>\delta
$$

Then from (\#),

$\epsilon_{k_{2}}\left|x_{2}^{\prime}\left(\sum_{j=n_{k_{1}+1}}^{n_{k_{2}}} t_{j}^{k_{2}} x_{j}\right)\right| \geq \epsilon_{k_{2}}\left|x_{2}^{\prime}\left(\sum_{j=1}^{n_{k_{2}}} t_{j}^{k_{2}} x_{j}\right)\right|-\epsilon_{k_{2}}\left|x_{2}^{\prime}\left(\sum_{j=1}^{n_{k_{1}}} t_{j}^{k_{2}} x_{j}\right)\right|>\delta-\delta / 2=\delta / 2$.

Continuing this construction produces an increasing sequence $\left\{k_{p}\right\},\left\{x_{p}^{\prime}\right\} \subset$ $B,\left\{t^{k_{p}}\right\} \subset A$ such that if $\left[n_{k_{p-1}+1}, n_{k_{p}}\right]=I_{p}$, then

$$
\text { (*) } \epsilon_{k_{p}}\left|x_{p}^{\prime}\left(\sum_{j \in I_{p}} t_{j}^{k_{p}} x_{j}\right)\right|>\delta / 2 \text {. }
$$

Define an infinite matrix

$$
M=\left[m_{p q}\right]=\left[\epsilon_{k_{p}} x_{p}^{\prime}\left(\sum_{j \in I_{q}} t_{j}^{k_{q}} x_{j}\right)\right] .
$$

We claim that $M$ is a signed- $\mathcal{K}$ matrix. First, the columns of $M$ converge to 0 since $\left\{x_{p}^{\prime}\right\}$ is $\sigma\left(X^{\prime}, X\right)$ bounded and $\epsilon_{k} \rightarrow 0$. Next, given any increasing sequence of integers, by the signed $\mathcal{F}$ GHP property there exist a further subsequence $\left\{r_{q}\right\}$ and signs $\left\{s_{q}\right\}$ such that $u=\sum_{q=1}^{\infty} s_{q} \chi_{I_{r_{q}}} t^{k_{r_{q}}} \in \lambda$. Then

$$
\begin{aligned}
\sum_{q=1}^{\infty} s_{q} m_{p r_{q}} & =\sum_{q=1}^{\infty} \epsilon_{k_{p}} x_{p}^{\prime}\left(s_{q} \sum_{j \in I_{r_{q}}} t_{j}^{k_{r_{q}}} x_{j}\right) \\
& =\epsilon_{k_{p}} x_{p}^{\prime}\left(\sum_{q=1}^{\infty} s_{q} \sum_{j \in I_{r_{q}}} t_{j}^{k_{r_{q}}} x_{j}\right)=\epsilon_{k_{p}} x_{p}^{\prime}\left(\sum_{q=1}^{\infty} u_{q} x_{q}\right)
\end{aligned}
$$

where $\sum_{q=1}^{\infty} u_{q} x_{q}$ is the $\sigma\left(X, X^{\prime}\right)$ sum of the series. Then $\sum_{q=1}^{\infty} s_{q} m_{p r_{q}} \rightarrow 0$ since $\epsilon_{p} \rightarrow 0$ and $\left\{x_{p}^{\prime}\right\}$ is $\sigma\left(X^{\prime}, X\right)$. By the Antosik-Mikusinski Matrix Theorem, the diagonal of $M$ converges to 0 . But, this contradicts $\left(^{*}\right)$ and the result follows.

A similar result under different assumptions is given in 2.6 of [9]. In particular, since the spaces $l^{\infty}$ and $m_{0}$ have the strong gliding hump property, the theorem covers the cases where the series are subseries or bounded multiplier convergent.

As the following example shows the Banach-Mackey assumption in Theorem 6 cannot be dropped. 
Example 7. Let $\lambda=c_{00}$ with the sup-norm and $X, X^{\prime}$ be any dual pair without the Banach-Mackey property. Let $\left\{x_{j}\right\} \subset X$ be weakly bounded but not strong bounded. Then the series $\sum x_{j}$ is $c_{00}$ multiplier convergent in any locally convex topology, but $\left\{e^{k}\right\}$ is $\|\cdot\|_{\infty}$ bounded and $\sum_{j=1}^{k} e_{j}^{k} x_{j}=$ $x_{k}$ so the partial sums of the series $\sum_{j=1}^{\infty} e_{j}^{k} x_{j}$ are not uniformly strong bounded. Note $c_{00}$ is monotone and, therefore, has WGHP.

\section{References}

[1] G. Bennett, A New Class of Sequence Spaces with Applications to Summability Theory, J. Reine Angew. Math., 266, pp. 49-75, (1974).

[2] J. Boos, Classical and Modern Methods in Summability, Oxford University Presss, Oxford, (2000).

[3] J. Boos, C. Stuart, C. Swartz, Gliding Hump Properties and Matrix Domains, Analysis Math., 30, pp. 243-257, (2004).

[4] P. K. Kamthan and M. Gupta, Sequence Spaces and Series, Marcel Dekker, N. Y., (1981).

[5] G. Köthe, Topological Vector Spaces I, Springer Verlag, Berlin, (1969).

[6] C. W. McArthur, On a Theorem of Orlicz and Pettis, Pacific J. Math., 22, pp. 297-303, (1967).

[7] D. Noll, Sequential Completeness and Spaces with the Gliding Humps Property, Manuscripta Math., 66, pp. 237-252, (1990).

[8] C. Stuart, Weak Sequential Completeness of $\beta$-Duals, Rocky Mountain Math. J., 26, pp. 1559-1568, (1996).

[9] C. Swartz, Multiplier Convergent Series, World Sci. Publ., Singapore, (2009).

[10] C. Swartz, An Abstract Gliding Hump Property, Proy.J. Math., 28, pp. 89-109, (2009). 
[11] A. Wilansky, Modern Methods in Topological Vector Spaces, McGrawHill, N. Y., (1978).

\section{Charles Swartz}

Department of Mathematics

New Mexico State University

Las Cruces, NM 88003,

U. S. A.

e-mail : cswartz@nmsu.edu 